\title{
Purification de la Protéine C-Réactive (CRP) par une procédure simplifiée en deux étapes chromatographiques
}

\author{
S. A. AKPONA ${ }^{1 *}$, M. GOMINA ASSOUMANOU $^{2}$, A .D. SEDOGBO ${ }^{4}$ \\ et I. ABDOULAYE ${ }^{3}$ \\ ${ }^{1}$ Professeur de Biochimie, UERS de Biochimie et Biologie Moléculaire, Faculté de Médecine, Université de \\ Parakou, BP 123 Parakou, Email : akponasimon@yahoo.fr (République du Bénin). \\ ${ }^{2}$ Assistant-Junior en Biochimie, UERS de Biochimie et Biologie Moléculaire, Faculté de Médecine, Université \\ de Parakou, BP 123 Parakou, Email : elboutraguero@yahoo.fr (République du Bénin). \\ ${ }^{3}$ Professeur agrégé de Biochimie, Faculté des Sciences de la Santé, BP 188 Cotonou, Université d'Abomey- \\ Calavi (République du Bénin). \\ ${ }^{4}$ Attachée de recherche, UERS de Biochimie et Biologie Moléculaire, Faculté de Médecine, Université de \\ Parakou, BP 123 Parakou (République du Bénin). \\ "Auteur correspondant, E-mail: akponasimon@yahoo.fr
}

\section{RESUME}

La purification de la Protéine C-Réactive (CRP) a été faite par une procédure simplifiée de chromatographie d'échange ionique en deux phases en tampons différents suivie d'une étape finale de Blue Sepharose à partir des sérums inflammatoires. Cette procédure a permis d'obtenir la protéine pure en peu de temps et de préparer son immun sérum monospécifique pour le diagnostic et le suivi des situations pathologiques en néonatologie, au cours de la réaction inflammatoire, ou d'autres pathologies de l'adulte pour lesquelles l'évaluation de cette protéine est d'une grande utilité.

(c) 2010 International Formulae Group. All rights reserved.

Mots clés: Chromatographie, immunoélectrophorèse, réaction inflammatoire.

\section{INTRODUCTION}

Décrite pour la première fois en 1930, la C-Reactive Protein (CRP) est aujourd'hui l'un des meilleurs marqueurs de la réaction inflammatoire (Mary et al., 2003 ; Okemefuna et al., 2010). Sa synthèse hépatique en réponse à un dommage tissulaire de type inflammatoire fait élever son taux plasmatique à plus de 100 fois la valeur sérique retrouvée chez l'individu normal (Bienvenu et al., 1985; Delmas et al., 2000). Un taux plasmatique de CRP élevé est corrélé avec une affection bactérienne (Rasamoelisoa et al., 1999 ; Mary et al., 2003 ; Simon et al., 2004 ; Casey et al., 2008), un risque cardiovasculaire accru (Panichi et al., 2000 ; Schwedler et al, 2006), et une mortalité élevée en soins intensifs (Kuvandik et al., 2007). Cela explique le grand intérêt que l'on attache à l'isolement de cette protéine en vue de la production d'immun sérum monospécifique à des fins diagnostiques et pour le suivi pronostique (Alt et al., 1982). La purification de la CRP était réalisée par des méthodes 
complexes et longues, associant de façon variable la chromatographie d'échange ionique, la chromatographie d'affinité, l'électrophorèse préparative, le comportement de la molécule en présence ou en absence du calcium (Ganrot et al., 1969; Johnson et Prellner, 1977 ; Kindmark et al., 1989). Dans ce travail, nous proposons une méthode simple, en deux étapes chromatographiques, pour la purification de la CRP à partir des sérums inflammatoires.

\section{MATERIEL ET METHODES Matériel}

Le sérum utilisé est un pool provenant de prélèvements effectués chez des patients opérés pour appendicite aigüe et au troisième jour post opératoire, dans le Service de Chirurgie Viscérale du Centre National Hospitalier et Universitaire de Cotonou (Benin).

Le DE23, le Blue Sepharose CL-6B et le gel d'agarose sont des produits Pharmacia. Le TRIS, l'acide chlorhydrique, le chlorure de sodium, le polyéthylène glycol 1500 (carbowax), l'EDTA et le chlorure de calcium sont des produits purs pour analyse. Ils ont servi à la préparation des différents tampons A et B utilisés dans les étapes de la purification.

\section{Méthodes}

Le DE23, un échangeur d'ions cellulosique, a servi à la première étape de séparation. Il a été gonflé et activé par un cycle acide-base intercalé de lavage à l'eau distillée avant d'être coulé dans une colonne de chromatographie Pharmacia $(2,5 \times 20 \mathrm{~cm})$. Il a été équilibré en tampon (Tris- $\mathrm{HCl} 40 \mathrm{mM}$, $\mathrm{CaCl}_{2} 5 \mathrm{mM}$, pH 7,4), tampon A, pendant une nuit à un débit de $20 \mathrm{ml} / \mathrm{h}$.

Le sérum inflammatoire $(25 \mathrm{ml})$ dialysé contre le tampon A a été déposé sur la colonne ainsi équilibrée et l'élution faite par le même tampon $(20 \mathrm{ml} / \mathrm{h})$ en collectant des fractions de $5 \mathrm{ml}$. Après l'élimination des fractions protéiques non fixées dans ces conditions, un gradient continu de $\mathrm{NaCl}, 0$ à $0,4 \mathrm{M}$, a permis de décrocher la fraction fixée sur la colonne. Cette fraction a été concentrée au carbowax et ramenée au volume initial de $25 \mathrm{ml}$ puis dialysée contre le tampon (Tris- $\mathrm{HCl} 40 \mathrm{mM}$, EDTA 5 mM, pH 7,4), tampon B. La fraction ainsi traitée a subi alors un deuxième passage chromatographique sur le gel DE23 régénéré et équilibré dans le tampon $\mathrm{B}$. Après élution de la fraction non fixée, les protéines fixées ont été décrochées en deux phases. La plupart des protéines contaminantes sont d'abord éliminées entre 0 et $0,4 \mathrm{M}$ de $\mathrm{NaCl}$. Ensuite, le reste des fractions fixées qui contenaient la totalité de la CRP de la prise d'essai a été élué entre 0,4 et $1 \mathrm{M}$.

A l'analyse (immunoélectrophorèse sur gel d'agarose à $0,8 \%$ ), cette fraction s'est révélée contenant de l'albumine qu'une étape de Blue Sepharose (deuxième étape chromatographique de séparation) en tampon (Tris- $\mathrm{HCl} 40 \mathrm{mM}, \mathrm{CaCl}_{2} 5 \mathrm{mM}, \mathrm{NaCl} 0,5 \mathrm{M}$, $\mathrm{pH} 7,4)$ a permis d'éliminer. La CRP a été enfin obtenue grâce à l'utilisation de 1,5 M $\mathrm{NaCl}$ (sur le gel Blue Sepharose) pour décrocher la presque totalité de la protéine à l'état pur.

La mise en évidence de protéines dans les fractions collectées a été faite par la lecture de l'absorbance au spectrophotomètre à 280 nm.

Le contrôle de la pureté de la préparation a été réalisé au moyen de l'immunoélectrophorèse standard et classique selon Grabar et Williams, et l'immunoélectrophorèse en deux dimensions (Briand et Paolaggi, 1997) avec, dans le gel d'agarose à $0,8 \%$, soit un immun sérum polyvalent, soit un anticorps monospécifique selon le cas. Enfin, un immun sérum anti-CRP a été préparé à partir de la protéine purifiée en utilisant le lapin fauve de race bourgogne comme animal de laboratoire.

\section{RESULTATS ET DISCUSSION}

Dans le présent travail, la CRP a été purifiée a partir des sérums humains 
inflammatoires par deux étapes chromatographiques, l'une d'échange ionique au DE23 et l'autre d'affinité au Blue Sepharose.

Le protocole que nous proposons dérive d'une analyse de plusieurs techniques en tenant compte des propriétés physicochimiques de la molécule de CRP. Il permet de partir de $25 \mathrm{ml}$ de sérum inflammatoire pour obtenir la CRP de pureté satisfaisante. La première étape de fractionnement du sérum inflammatoire sur le gel DE23 a été réalisée en deux phases chromatographiques. La première phase a permis d'obtenir le profil d'élution de la Figure 1. L'immunoélectrophorèse sur gel d'agarose réalisée sur les fractions recueillies, avec le sérum humain normal comme témoin, a permis de situer la CRP dans la fraction fixée sur le gel DE23 et décrochée par le gradient de $\mathrm{NaCl}$ 0-0,4 M (Figure 4).

La fraction décrochée par le gradient $\mathrm{NaCl} 0-0,4 \mathrm{M}$ issue de la phase précédente a fait l'objet d'une deuxième phase chromatographique sur le gel DE23 dans un tampon différent (tampon B). En dehors de la fraction non fixée dans ces conditions, un gradient de $\mathrm{NaCl}$ de 0 à $0,4 \mathrm{M}$ a permis d'éliminer l'ensemble des autres protéines fixées qui souillent la CRP. La protéine Créactive qui, dans nos conditions de travail restait encore solidement fixée sur le gel a été décrochée par un gradient de $\mathrm{NaCl}$ de 0,4 à 1 M (Figure 2).

L'analyse de cette fraction par immunoélectrophorèse sur gel d'agarose présumée ne contenir que la CRP à l'état pure s'est révélée encore contaminée par de l'albumine (Figure 5). Cette situation nous a obligé à recourir à une dernière étape de chromatographie sur une colonne de Blue Sepharose en tampon (Tris- $\mathrm{HCl} 40 \mathrm{mM}$, $\mathrm{CaCl} 25 \mathrm{mM}, \mathrm{NaCl} 0,5 \mathrm{M}, \mathrm{pH} 7,4)$. Ces conditions ont modifié le comportement du gel vis-à-vis de l'albumine qui, au lieu d'être retenu, est sortie presque en totalité. La CRP fixée sur le gel a été alors décrochée avec une molarité de 1,5 $\mathrm{M}$ en $\mathrm{NaCl}$ (Figures 3 et 6).

Les Figures 7 et 8 montrent une pureté satisfaisante de la préparation de la CRP selon notre protocole de travail.

L'immun sérum préparé à partir de cette protéine ainsi purifiée est monospécifique comme l'indique le contrôle immunoélectrophorétique sur gel d'agarose (Figure 9).

Les stratégies mises en œuvre pour la purification de la CRP dépendent des paramètres comme, entre autres, sa concentration, sa susceptibilité à être précipitée par les sels, son interaction avec certains sucres, son comportement physicochimique dans certains milieux (Fujise et al., 1992 ; Kindmark et al., 1989 ; Pruden et al, 1988 ; Sarikaputi et al., 1991 ; Takiguchi et al., 1990 ; Wu et al, 2003). Les anticorps monoclonaux n'ont pas été épargnés dans cette entreprise délicate de purification de la CRP. Comme le décrivent Yamamoto et al. (1992), les étapes de purification les plus couramment utilisées comportent une à deux phases de chromatographie d'échange ionique (Ganrot et al., 1969; Johnson et Prellner, 1977), une phase de chromatographie d'affinité associant, en fin de procédure, une électrophorèse préparative qui fait appel au comportement de la molécule en présence ou en absence du calcium dans le tampon de séparation (Kindmark et al., 1989; Parra et al., 2005). Toutes ces méthodes sont longues, lourdes et onéreuses. Le protocole que nous présentons dans ce travail part d'une faible quantité de sérum inflammatoire, $25 \mathrm{ml}$, passe par une étape d'échange ionique en deux temps avec deux systèmes tampon différents et une étape de Blue Sepharose pour obtenir la molécule de CRP à l'état pur. Il épargne des techniques d'immunoaffinité et d'électrophorèse préparative indispensables dans les différents protocoles conventionnels. Enfin, il 


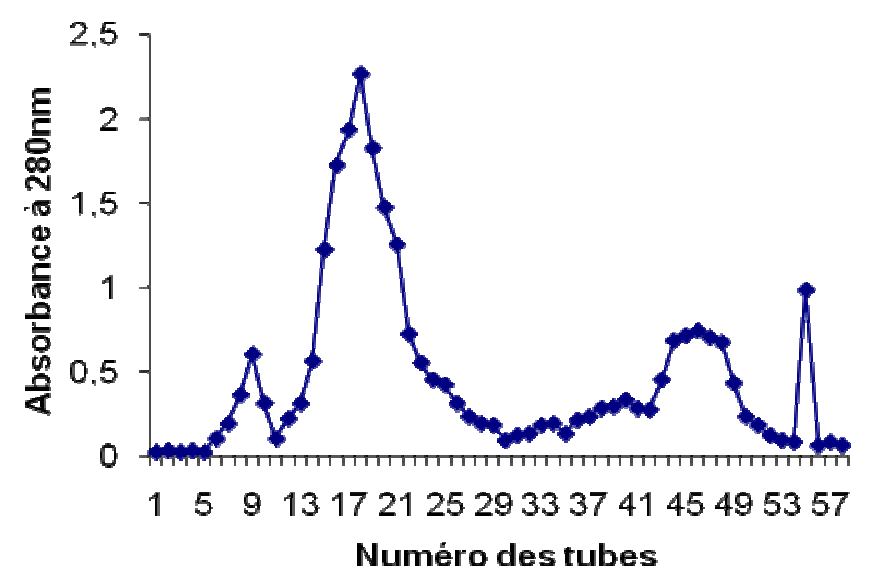

A

B

Figure 1: Profil d'élution des protéines de la fraction inflammatoire sur le gel DE23 ( $1^{\mathrm{er}}$ passage). A-Tampon Tris-HCl 40 mM, $\mathrm{CaCl}_{2} 5 \mathrm{mM}$, pH 7,4; B- Gradient $\mathrm{NaCl} 0-0,4 \mathrm{M}$.

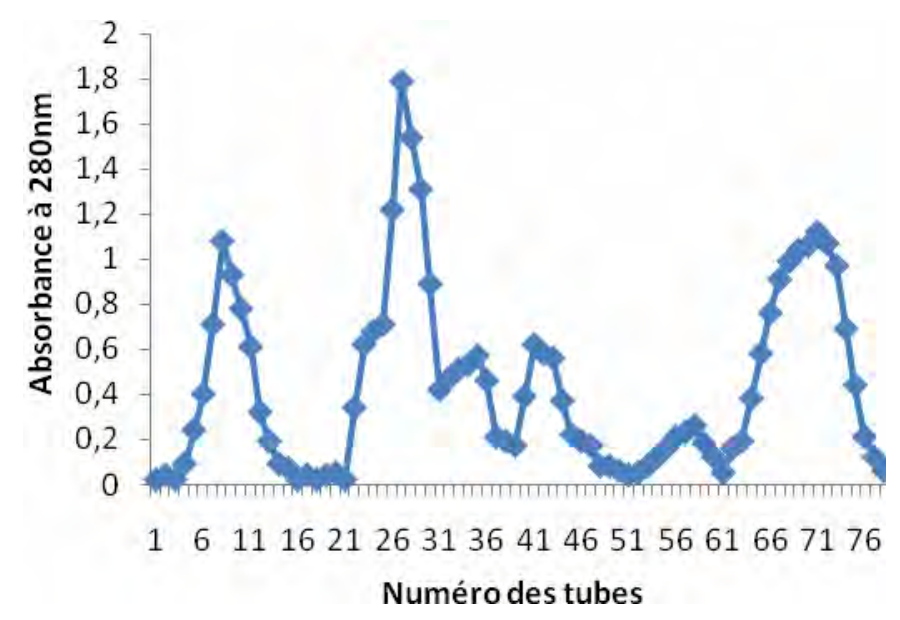

A

B

$\mathrm{C}$

Figure 2: Profil d'élution des protéines de la fraction inflammatoire sur le gel DE23 ( $2^{\text {ème }}$ passage). A-Tampon Tris-HCl 40 mM, EDTA 5 mM, pH 7,4 ; B- Gradient NaCl 0-0,4 M ; C- Gradient NaCl 0,4-1 M. 


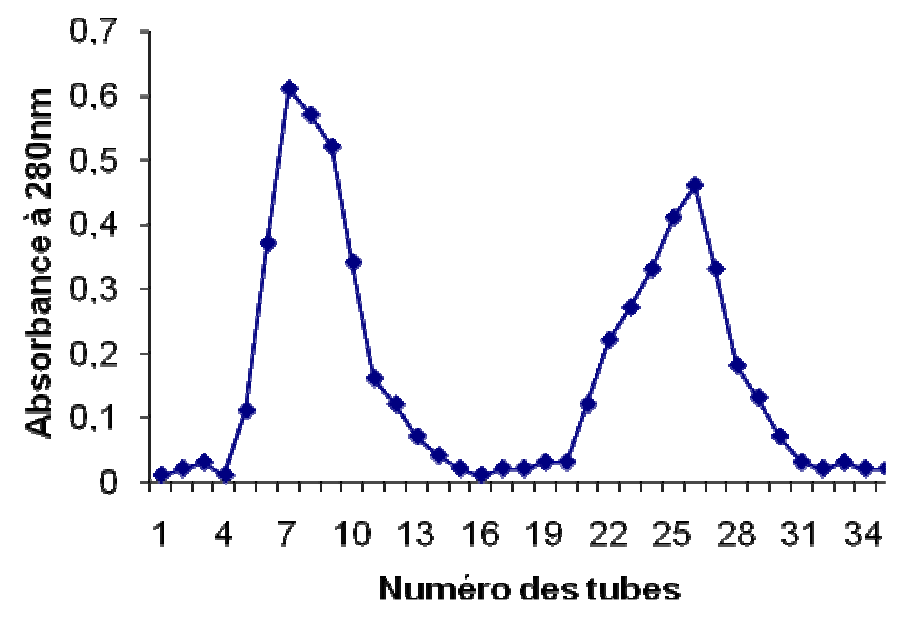

A

B

Figure 3 : Profil d'élution des protéines de la fraction inflammatoire sur le gel Blue sepharose.

A-Tampon Tris- $\mathrm{HCl} 40 \mathrm{mM}, \mathrm{CaCl}_{2} 5 \mathrm{mM}, \mathrm{NaCl} 0,5 \mathrm{M}, \mathrm{pH}$ 7,4; B- Tampon Tris- $\mathrm{HCl} 40 \mathrm{mM}, \mathrm{CaCl}_{2} 5 \mathrm{mM}, \mathrm{NaCl} 1,5 \mathrm{M}$, $\mathrm{pH} 7,4$.

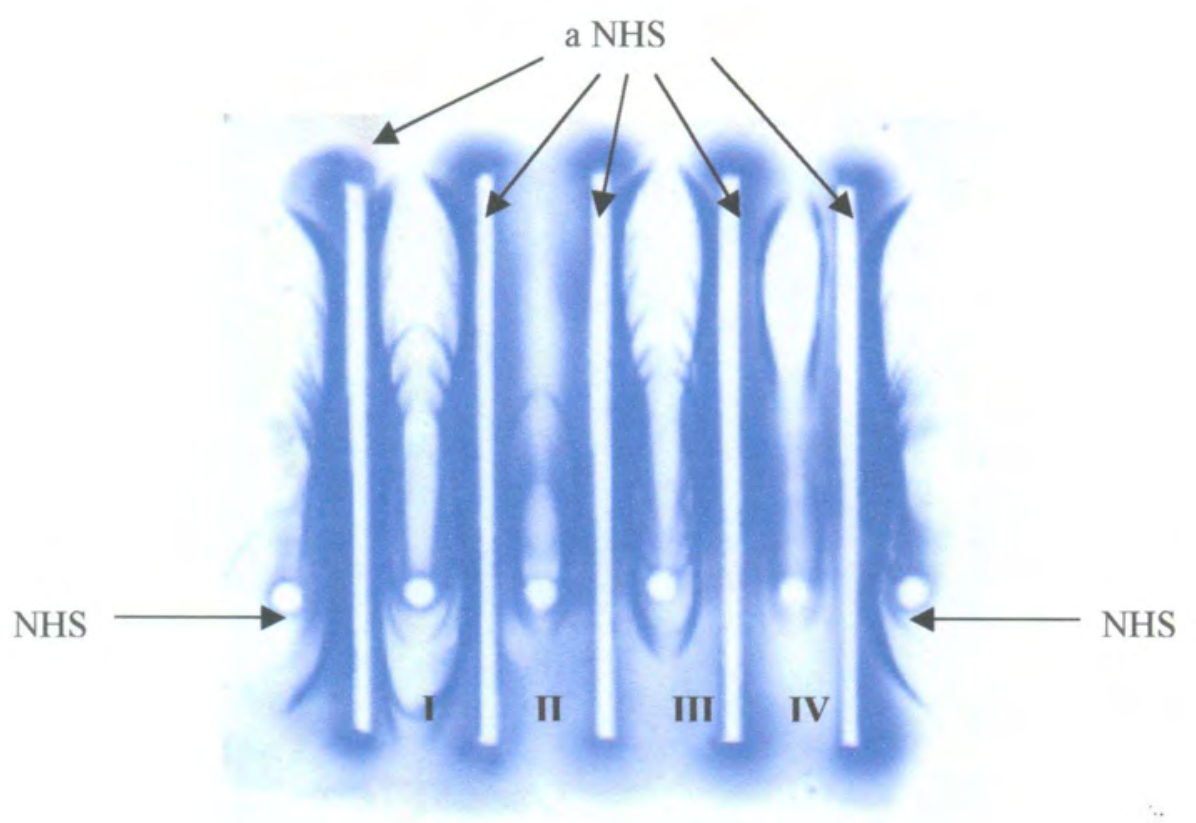

Figure 4 : Immunoélectrophorégramme sur gel d'agarose des fractions obtenues lors du $1^{\text {er }}$ passage sur le gel DE23.

I- Fraction inflammatoire déposée sur le gel DE23 ; II- Fraction non fixée sur le gel DE23 ; III et IV- Fraction décrochée avec un gradient $\mathrm{NaCl} 0-0,4 \mathrm{M}$.

NHS : Sérum humain normal ; aNHS : anticorps anti-NHS. 


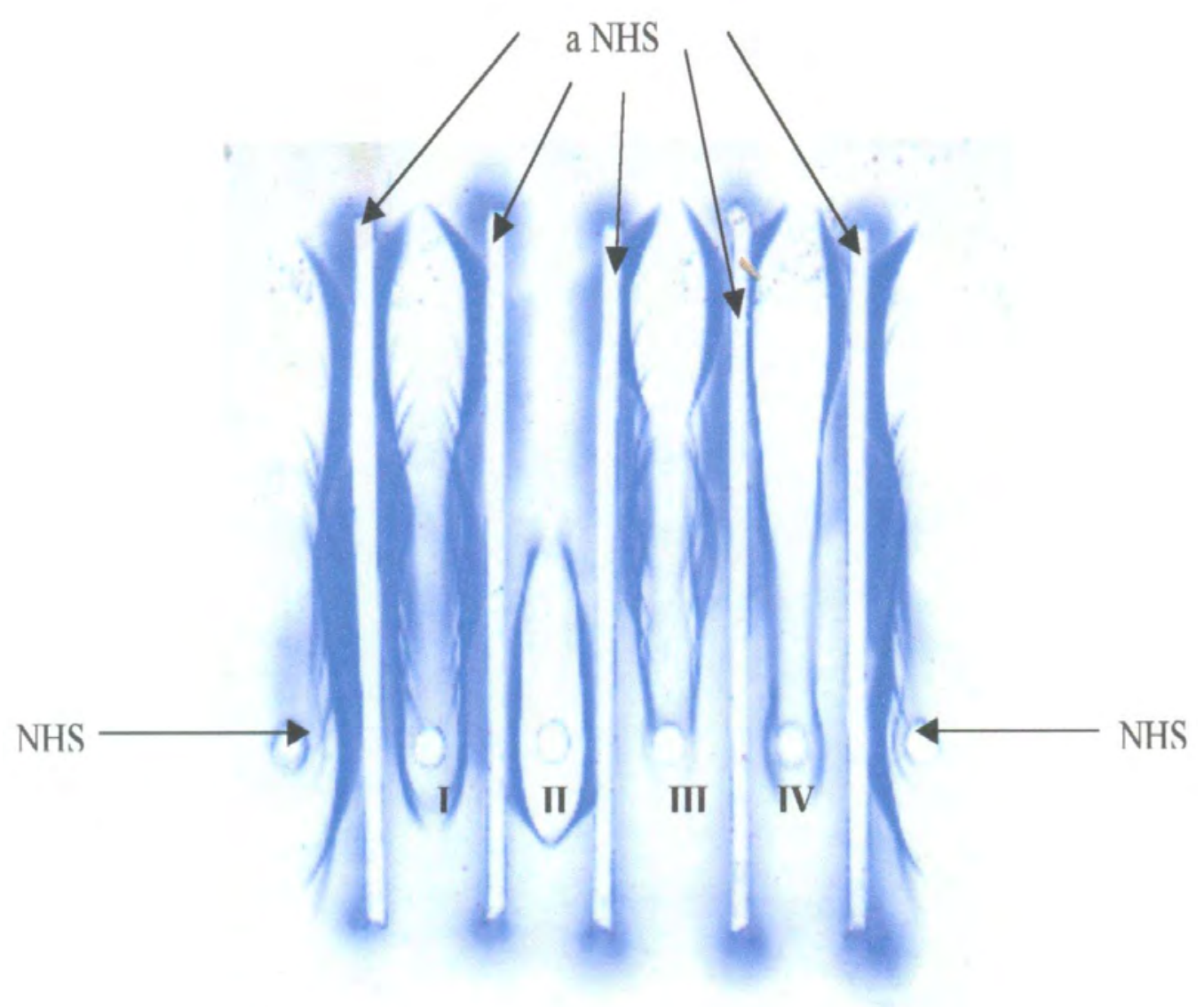

Figure 5 : Immunoélectrophorégramme sur gel d'agarose des fractions obtenues lors du $2^{\text {ème }}$ passage sur le gel DE23.

I- Fraction décrochée par $\mathrm{NaCl} 0-0,4 \mathrm{M}$ lors du $1^{\mathrm{er}}$ passage sur le gel DE23 ; II- Fraction non fixée sur le gel DE23 ; IIIFraction décrochée avec un gradient $\mathrm{NaCl}$ 0-0,4 M ; IV- Fraction décrochée avec un gradient $\mathrm{NaCl}$ 0,4-1 M. NHS : Sérum humain normal ; aNHS : anticorps anti-NHS.

est rapide et pratique pour obtenir la CRP dont le rôle biologique très important n'est pas encore totalement exploré. La protéine Créactive (CRP) est une protéine d'une grande importance en biologie clinique. De ce fait, purifier la CRP et disposer de son immun sérum monospécifique devient une entreprise utile en biologie clinique à des fins diagnostiques et pour le suivi. En effet, en association avec l'orosomucoïde, la CRP permet de faire le diagnostic du début de l'infection puis d'affirmer la guérison lorsque le taux sérique des deux protéines est revenu à la normale.

Bien que nous n'ayons pas pris en compte la question du rendement au cours de la purification, la technique décrite a permis d'obtenir la CRP à l'état pur. Cette préparation injectée au lapin fauve de race bourgogne permet d'obtenir un immun sérum anti-CRP monospécifique utilisable en laboratoire pour la mise au point de diverses méthodes immunochimiques. 


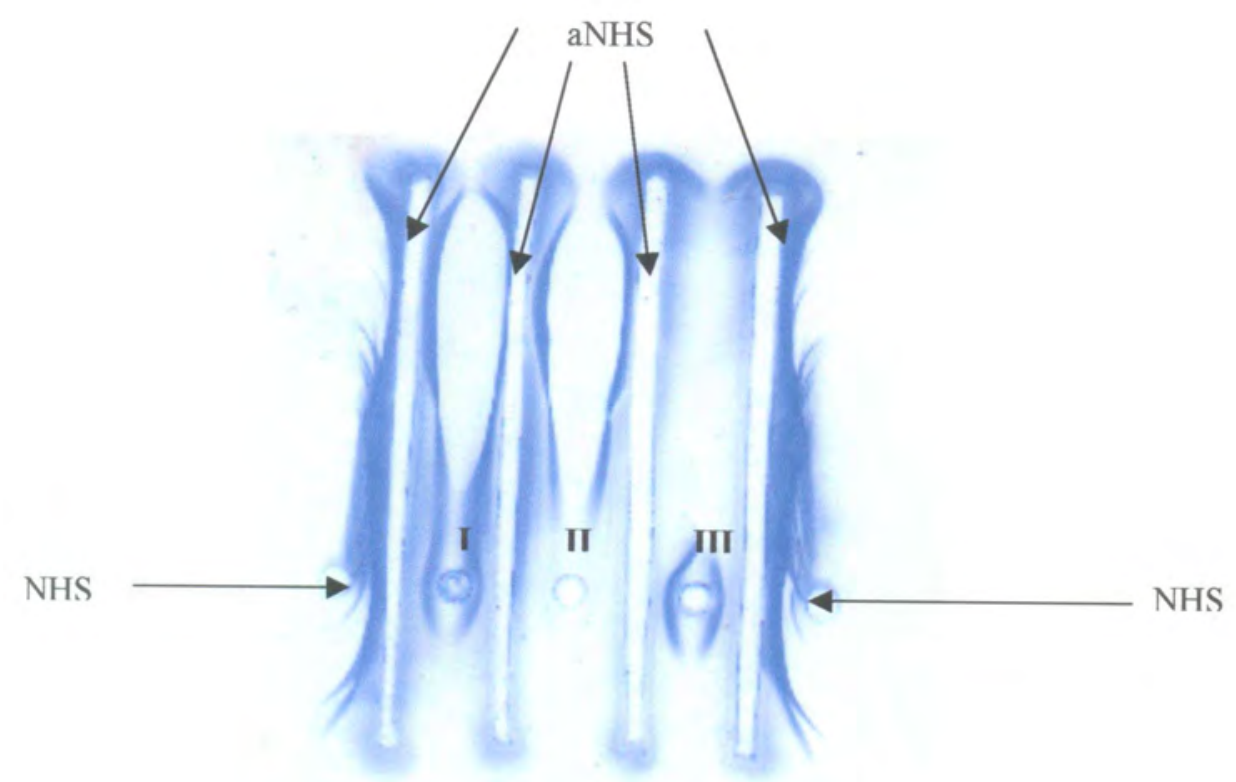

Figure 6 : Immunoélectrophorégramme sur gel d'agarose des fractions obtenues lors du passage sur le gel Blue Sepharose.

I- Fraction décrochée avec un gradient $\mathrm{NaCl}$ 0,4-1 M sur le gel DE23 ; II- Fraction décrochée avec $\mathrm{NaCl} 0,5 \mathrm{M}$ sur le gel Blue Sepharose ; III- Fraction décrochée avec NaCl 1,5 M sur le gel Blue Sepharose. NHS : Sérum humain normal ; aNHS : anticorps anti-NHS.

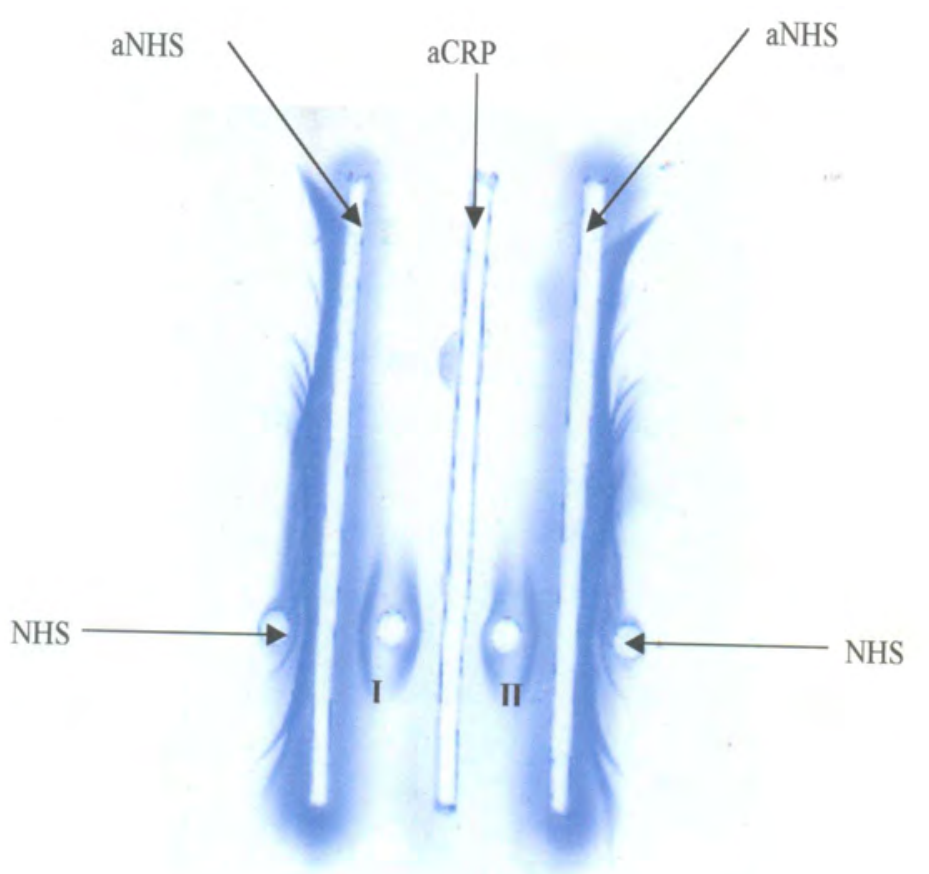

Figure 7 : Immunoélectrophorégramme sur gel d'agarose de la fraction décrochée avec $\mathrm{NaCl}$ 1,5 M sur le gel Blue Sepharose.

I et II- Fraction décrochée avec $\mathrm{NaCl}$ 1,5 M sur le gel Blue Sepharose.

NHS : Sérum humain normal ; aNHS : anticorps anti-NHS ; aCRP : anticorps anti-CRP du commerce. 


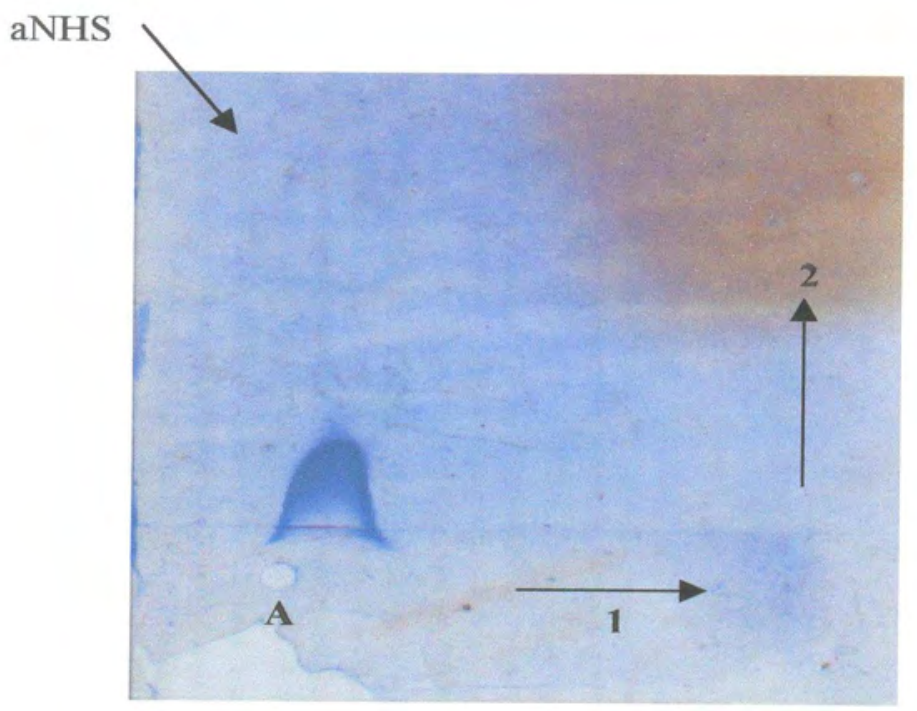

Figure 8 : Immunoélectrophorégramme double dimension sur gel d'agarose de la fraction décrochée avec $\mathrm{NaCl}$ 1,5 M sur le gel Blue Sepharose.

$1-1^{\text {er }}$ sens de migration électrophorétique ; 2 - $2^{\text {ème }}$ sens de migration électrophorétique. aNHS : anticorps anti-NHS.

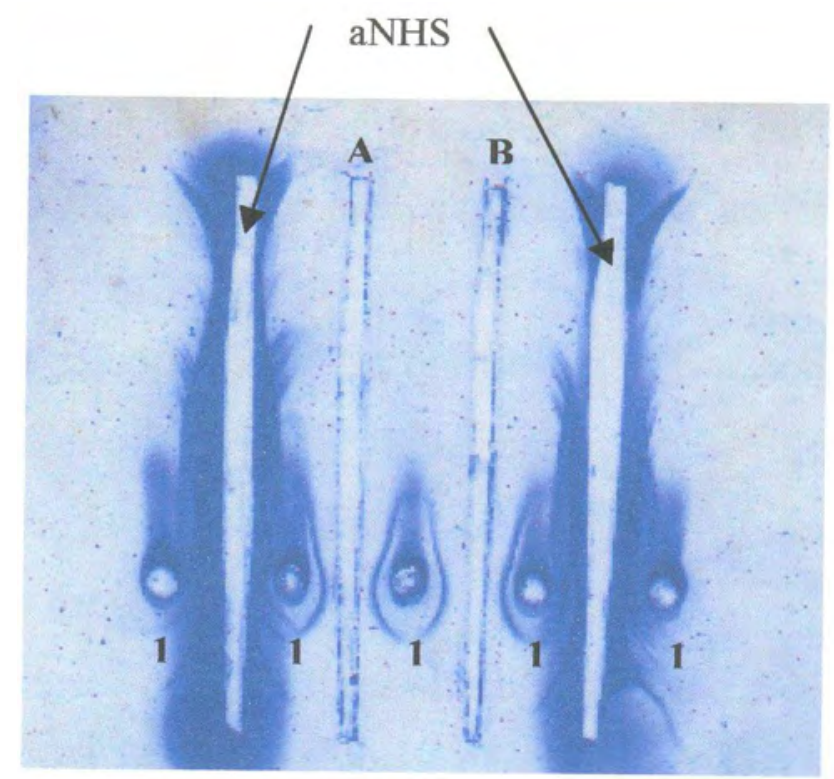

Figure 9 : Immunoélectrophorégramme de contrôle de pureté de la CRP contenue dans la fraction décrochée avec $\mathrm{NaCl}$ 1,5 M sur le gel Blue Sepharose.

aNHS : anticorps anti-NHS.

A-Anticorps anti-CRP de commerce.

B- Anticorps anti-CRP préparés chez le lapin à partir de la CRP purifiée. 


\section{RÉFÉRENCES}

Alt R, Willard D, Messer J, Metais P, Goester C, Mark JJ. 1982. Intérêt de la C-réactive protéine dans les infections bactériennes néonatales. Arch. Fr. Pédiatr., 39: 811813.

Bienvenu J, Bienvenu F, Baltassat P. 1985. Profil protéique du nouveau-né : intérêt particulier des protéines de l'inflammation. Le Pharmacien Biologiste, 19(156) : 101/31-105/35.

Briand P, Paolaggi F. 1997. Méthodes électrophorétiques et électrochimiques. In Appareils et Méthodes en Biochimie et Biologie Moléculaire, Kamoun P (ed). Edition Flammarion Médecine-Science : Paris ; 213-230.

Casey R, Newcombe J, McFadden JJ, Bodman-Smith KB. 2008. The acute phase reactant $\mathrm{C}$-reactive protein binds to phosphorylcholin-expressing Neisseria meningitides and inceases uptake by human phagocytes. Infection and Immunity, 76(3): 1298-1304.

Delmas J, Monneret G, Lapillonne A, Basson $\mathrm{E}$, Isaac $\mathrm{C}$, Bienvenu $\mathrm{F}$, Putet $\mathrm{G}$, Bienvenu J. 2000. Cinétiques de la prolactine et de la C-réactive protéine dans les infections néonatales à streptocoque du groupe B. Annales de Biologie Clinique, 58(2): 208-211.

Fujise H, Takanami H, Yamamoto M, Ohta I, Yamamoto $S$, Fukase $T$, Naiki $M$, Akihama S, Ogawa E, Takahashi R. 1992. Simple isolation of canine C reactive protein (CRP) by phosphorylcholine (PC) affinity chromatography. J. Vet. Med. Sci., 54(1): 165-167.

Ganrot PO, Kindmark CO. 1969. A simple two-step procedure for isolation of C reactive protein. Biochem Biophys Acta, 194: 443-448.

Johnson U, Prellner K. 1977. Purification of C-reactive protein on DEAE-Cellulose by a simple two-step procedure utilizing the calcium dependency of the protein.
Biochemica and Biophysica Acta, 495: 349-353.

Kindmark C-O L, Williams JC. 1989. Purification of human C-reactive protein by barium sulfate and preparative agarose electrophoresis. A PMIS, 97: 891-896.

Kuvandik G, Ucar E, Borazan A, Aydemir S, Ilikhan S, Sekitmez N, Duru M, Ozer B, Kaya H. 2007. Markers of inflammation as determinants of mortality in intensive care unit patients. Advances in Therapy, 24(5): 1078-1084.

Mary R, Veinberg F, Couderc R. 2003. Les méningites aigües, protéines inflammatoires et procalcitonine. Annales de Biologie Clinique, 61(2): 127-137.

Okemefuna AI, Stach L, Rana S, Ziai Buetas AJ, Gor J, Perkins ST. 2010. C-reactive protein exists in $\mathrm{NaCl}$ concentrationdependant pentamer-decamer equilibrium in physiological buffer. J.B.C., $\mathbf{2 8 5}$ : 1041-1052.

Panichi V, Migliori M, De Pietro S, Taccola D, Andreini B, Metelli MR, Giovannini L, Palla R. 2000. The link of biocompatibility to cytokine production. Kidney International, 58: 96-103.

Parra MD, Mika Tuomola DVM, CabezasHerrera J, Ceron JJ. 2005. Use of a time resolve immunofluorimetric assay for determination of canine C-reactive protein concentrations in whole blood. Am. J. Vet. Res., 66: 62-66.

Pruden JD, Connolly M.K, Stecher JV. 1988. Single-step purification of rat $\mathrm{C}$ - reactive protein and generation of monospecific C-reactive protein antibody. $J$. Chromatography, 437: 399-410.

Rasamoelisoa JM, Tovone XG, Andriamandy RCL, Rasamoela NW, Rasamindrakotroka A. 1999. Intérêt de la C-reactive protein (CRP) dans les affections fébriles de l'enfant. Arch. Inst. Pasteur Madagascar, 65(2):113-116.

Sarikaputi M, Morimatsu M, Syuto B, Saito M, Naiki M. 1991. A new purification procedure for bovine C-reactive protein 
and serum amyloid $\mathrm{P}$ component. $J$. Biochem., 23(10): 1137-1142.

Schwedler SB, Filep JG, Galle J, Wanner C, Potempa LA. 2006. C-reactive protein: a family of proteins to regulate cardiovascular function. Am. J. Kidney Dis., 47: 212-222.

Simon L, Gauvin F, Amre DK, Saint-Loui P, Lacroix J. 2004. Serum procalcitonin and C-reactive protein levels as markers of bacterial infection: a systematic review and meta analysis. Clinical Infectious Diseases, 39: 206-217.
Takiguchi M, Fujinaga T, Naiki M, Mizuno S, Otomo K. 1990. Isolation, characterisation and quantitative analysis of $\mathrm{C}$ reactive protein from horses. Am. J. Vet. Res., 51(8): 1215-1220.

Wu Y, Xang Hw, Ji SR, Sui S. 2003. Two dimensional cristallization of rabbit Creactive protein monomeric subunit. Acta Crist., 59: 922-926.

Yamamoto S, Tagata $\mathrm{K}$, Nagahata $\mathrm{H}$, Ishikawa Y, Morimatsu M, Naiki M. 1992. Isolation of canine C-reactive protein and characterization of its properties. Vet. Immunol. Immunopathology, 30: 329-339. 